This item was submitted to Loughborough's Research Repository by the author.

Items in Figshare are protected by copyright, with all rights reserved, unless otherwise indicated.

\title{
Quasicrystalline order and a crystal-liquid state in a soft-core fluid
}

PLEASE CITE THE PUBLISHED VERSION

http://dx.doi.org/10.1103/PhysRevLett.111.165501

PUBLISHER

(C) American Physical Society

VERSION

VoR (Version of Record)

LICENCE

CC BY-NC-ND 4.0

REPOSITORY RECORD

Archer, Andrew J., Alastair M. Rucklidge, and Edgar Knobloch. 2019. "Quasicrystalline Order and a Crystalliquid State in a Soft-core Fluid”. figshare. https://hdl.handle.net/2134/15351. 
This item was submitted to Loughborough's Institutional Repository (https://dspace.lboro.ac.uk/) by the author and is made available under the following Creative Commons Licence conditions.

\section{creative
commons}

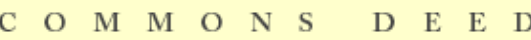

Attribution-NonCommercial-NoDerivs 2.5

You are free:

- to copy, distribute, display, and perform the work

Under the following conditions:

Attribution. You must attribute the work in the manner specified b the author or licensor.

Noncommercial. You may not use this work for commercial purposes.

No Derivative Works. You may not alter, transform, or build upon this work.

- For any reuse or distribution, you must make clear to others the license terms of this work.

- Any of these conditions can be waived if you get permission from the copyright holder.

Your fair use and other rights are in no way affected by the above.

This is a human-readable summary of the Leqal Code (the full license).

\section{Disclaimer 만}

For the full text of this licence, please go to: http://creativecommons.org/licenses/by-nc-nd/2.5/ 


\title{
Quasicrystalline Order and a Crystal-Liquid State in a Soft-Core Fluid
}

\author{
A. J. Archer, ${ }^{1}$ A. M. Rucklidge, ${ }^{2}$ and E. Knobloch ${ }^{3}$ \\ ${ }^{1}$ Department of Mathematical Sciences, Loughborough University, Loughborough LE11 3TU, United Kingdom \\ ${ }^{2}$ Department of Applied Mathematics, University of Leeds, Leeds LS2 9JT, United Kingdom \\ ${ }^{3}$ Department of Physics, University of California at Berkeley, Berkeley, California 94720, USA
}

(Received 9 April 2013; published 15 October 2013)

\begin{abstract}
A two-dimensional system of soft particles interacting via a two-length-scale potential is studied. Density functional theory and Brownian dynamics simulations reveal a fluid phase and two crystalline phases with different lattice spacing. Of these the larger lattice spacing phase can form an exotic periodic state with a fraction of highly mobile particles: a crystal liquid. Near the transition between this phase and the smaller lattice spacing phase, quasicrystalline structures may be created by a competition between linear instability at one scale and nonlinear selection of the other.
\end{abstract}

DOI: 10.1103/PhysRevLett.111.165501

Crystals are ordered arrangements of atoms or molecules with rotation and translation symmetries. Quasicrystals (QCs), discovered in 1982 [1], lack the lattice symmetries of crystals and yet have discrete Fourier spectra. QCs have been found not only in metals but also in colloidal systems [2,3], mesoporous silica [4], and softmatter systems [5]. The latter can form micelles [6,7], e.g., from dendrimers or block copolymers, comprising a hydrophobic polymer core surrounded by a corona of flexible hydrophilic polymer chains. Theoretical approaches to investigating the stability of metallic or micellar QCs often involve minimizing an appropriate energy, but the principle underlying their stability is not known $[8,9]$.

Patterns with quasicrystalline structure, or quasipatterns, were discovered in Faraday wave experiments in the 1990s; two mechanisms for stabilizing these were identified [10]. The first, relevant to experiments in Ref. [11], involves one length scale and may lead to a stable quasipattern [12]. The second, involving coupling between an unstable scale and weakly damped (or weakly excited) waves with a different length scale, is relevant to the experiments in Refs. [13-16], and was explored in [17-22]. This mechanism can also operate for soft-matter QCs [23-26]. Here, we observe a dynamic mechanism for forming QCs involving two length scales that is qualitatively different: the system first forms a small length scale crystal. Only when this phase is almost fully formed (i.e., the dynamics is far into the nonlinear regime) does the longer length scale start to appear, leading to the formation of QCs. This process occurs in a region of the phase diagram where the linear growth of density fluctuations in a quenched uniform fluid selects the shorter scale but nonlinear stability favors a longer scale.

The effective coarse-grained interaction potentials between the centres of mass of polymers, dendrimers, or other such macromolecules are soft. By this we mean that they are finite for all separation distances $r$, because the center of mass of such soft objects does not necessarily
PACS numbers: 61.50.Ah, 05.20.-y, 61.44.Br, 64.70.D-

coincide with any individual monomer. The soft effective pair potential between such particles can be approximated as $V(r)=\epsilon e^{-(r / R)^{n}}$. Simple linear polymers in solution correspond to the case $n=2$ with the length $R$ of order the radius of gyration and the energy $\epsilon$ for a pair of polymers to fully overlap of order $2 k_{B} T$, where $k_{B}$ is Boltzmann's constant and $T$ is the temperature [27-33]. Dendrimers, due to the nature of their chemical architecture, can have an effective interaction with a higher value of $n$; such systems form so-called cluster crystals [33] and there has been much interest in soft potential models for these systems [34-40].

Here we consider a model two-dimensional system of soft particles that interact via the potential

$$
V(r)=\epsilon e^{-(r / R)^{8}}+\epsilon a e^{-\left(r / R_{s}\right)^{8}} .
$$

This potential is finite for all $r$ and has a shoulder when the parameter $a \neq 0$, with two length scales. The radius of the core is $R$ and the radius of the shoulder is $R_{s}>R$; the energy for complete overlap is $(1+a) \epsilon$. Such a potential is a simple coarse-grained model for the effective interaction between dendrimers, star polymers, or micelles formed, e.g., from block copolymers, which have a stiff hydrophobic core surrounded by a corona of flexible hydrophilic chains. A related, but piecewise constant potential is used in Ref. [25]. The limits (i) $a \rightarrow 0$ or (ii) $a \rightarrow \infty$ and $\epsilon \rightarrow 0$ with $\epsilon a=$ const both result in systems with a single crystal phase. In the following we set the dimensionless interaction energy parameter $\beta \epsilon=1$, where $\beta=\left(k_{B} T\right)^{-1}$, and fix the ratio of the two length scales to be $R_{s} / R=1.855$ (see below).

We use density functional theory (DFT) [41-43] to study this system. The grand free energy is

$$
\begin{aligned}
\Omega[\rho(\mathbf{r})]= & k_{B} T \int d \mathbf{r} \rho(\mathbf{r})\left[\ln \Lambda^{2} \rho(\mathbf{r})-1\right]+\mathcal{F}_{\mathrm{ex}}[\rho(\mathbf{r})] \\
& +\int d \mathbf{r}[\Phi(\mathbf{r})-\mu] \rho(\mathbf{r}),
\end{aligned}
$$


which is a functional of the one-body (number) density of the particles, $\rho(\mathbf{r})$, where $\mathbf{r}=(x, y)$. The first term is the ideal-gas contribution to the free energy $\mathcal{F}_{\text {id }}, \Lambda$ is the thermal de Broglie wavelength, $\mu$ is the chemical potential, $\Phi(\mathbf{r})$ is any external potential that may be confining the system, and $\mathcal{F}_{\text {ex }}[\rho(\mathbf{r})]$ is the excess Helmholtz free energy from the interactions between the particles. The equilibrium density profile is that which minimizes $\Omega[\rho(\mathbf{r})]$; the corresponding minimum is the thermodynamic grand potential of the system. For a system in the bulk fluid state [i.e., where $\Phi(\mathbf{r}) \equiv 0$ ], the minimizing density is uniform, $\rho=\rho_{0}$. However, for other state points, when the system freezes to form a solid, $\Omega$ is minimized by a nonuniform $\rho(\mathbf{r})$, exhibiting sharp peaks. For the systems of soft-core particles considered here, one may approximate $\mathcal{F}_{\text {ex }}$ as [27]

$$
\mathcal{F}_{\text {ex }}[\rho(\mathbf{r})]=\frac{1}{2} \int d \mathbf{r} \int d \mathbf{r}^{\prime} \rho(\mathbf{r}) V\left(\left|\mathbf{r}-\mathbf{r}^{\prime}\right|\right) \rho\left(\mathbf{r}^{\prime}\right) .
$$

This functional generates the random phase approximation (RPA) for the pair direct correlation function $c^{(2)}\left(\mathbf{r}, \mathbf{r}^{\prime}\right) \equiv$ $-\beta \frac{\delta^{2} \mathcal{F}_{\mathrm{ex}}}{\delta \rho(\mathbf{r}) \delta \rho\left(\mathbf{r}^{\prime}\right)}=-\beta V\left(\left|\mathbf{r}-\mathbf{r}^{\prime}\right|\right)[41-43]$. If we assume that these are Brownian particles with dynamics

$$
\dot{\mathbf{r}}_{i}=-\Gamma \nabla_{i} U\left(\left\{\mathbf{r}_{i}\right\}, t\right)+\Gamma \mathbf{X}_{i}(t),
$$

where the index $i=1, \ldots, N$ labels particles, $U\left(\left\{\mathbf{r}_{i}\right\}, t\right)=$ $\sum_{i=1}^{N} \Phi\left(\mathbf{r}_{i}\right)+\sum_{i \neq j} V\left(\mathbf{r}_{i}-\mathbf{r}_{j}\right)$ is the potential energy of the system and $\mathbf{X}_{i}(t)$ is a white noise term, we can investigate the dynamics of the system using dynamic density functional theory (DDFT) [44-47] in the form

$$
\frac{\partial \rho(\mathbf{r}, t)}{\partial t}=\Gamma \nabla \cdot\left[\rho(\mathbf{r}, t) \nabla \frac{\delta \Omega[\rho(\mathbf{r}, t)]}{\delta \rho(\mathbf{r}, t)}\right]
$$

where $\rho(\mathbf{r}, t)$ is now the time-dependent nonequilibrium one-body density profile and $\Gamma \equiv \beta D$ is the mobility. Here $D$ is the diffusion coefficient. In deriving Eq. (5) we have used the equilibrium free energy $\mathcal{F}=\mathcal{F}_{\text {id }}+\mathcal{F}_{\text {ex }}$ to approximate the unknown nonequilibrium free energy.

Figure 1(a) shows the equilibrium phase diagram calculated using Picard iteration [48] of the DFT EulerLagrange equation, starting either from the profile for a nearby state point or a uniform density profile with a small random value added to each point. As the fluid density is increased, the system freezes to form one of two distinct solid phases (Fig. 2): for larger values of $a$ the system forms crystal $A$, a hexagonal crystal with a large lattice spacing, but for smaller values of $a$ it forms crystal $B$, a hexagonal crystal with a much smaller lattice spacing. The red regions in Fig. 1(a) denote thermodynamic coexistence between two different phases at the same temperature, pressure, and chemical potential.

To understand the phase diagram we study the structure and stability of a uniform liquid with density $\rho_{0}$ and $\Phi(\mathbf{r}) \equiv$ 0 . We follow [41,46,49,50] and expand Eq. (5) in powers of $\tilde{\rho}(\mathbf{r}, t) \equiv \rho(\mathbf{r}, t)-\rho_{0}$. Retaining only linear terms, we find that the growth or decay of different Fourier modes of wave
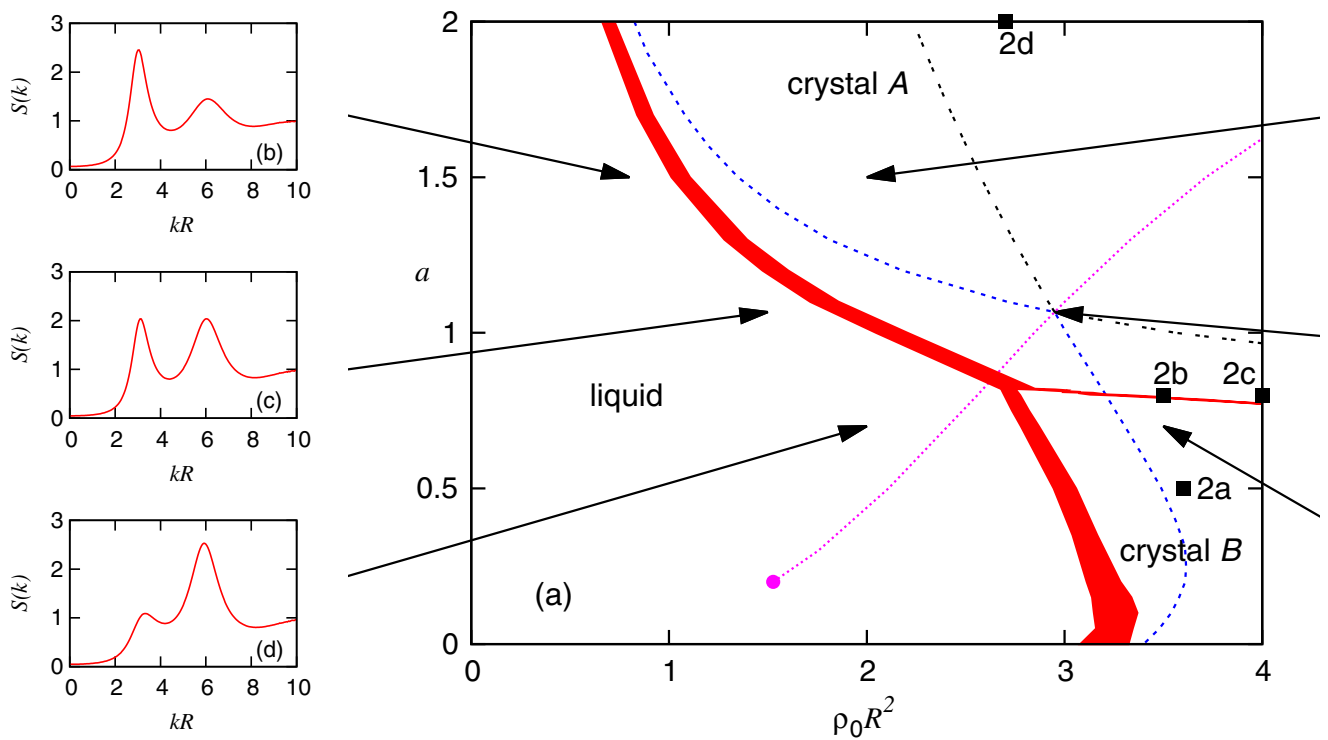
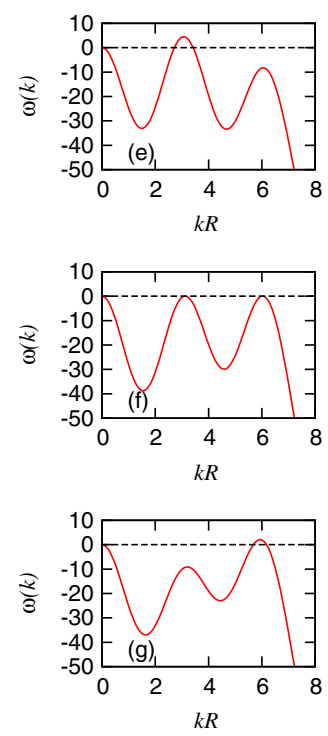

FIG. 1 (color online). Phase diagram, static structure factor $S(k)$, and dispersion relation $\omega(k)$ for $\beta \epsilon=1$ and $R_{s} / R=1.855$. (a) The bulk system phase diagram in the $\left(\rho_{0} R^{2}, a\right)$ plane. The system exhibits a uniform fluid phase and two crystal phases: the larger lattice spacing crystal $A$ phase and the smaller lattice spacing crystal $B$ phase. The regions filled in red denote areas where there is two-phase coexistence between the different phases. The blue dashed line denotes the linear instability threshold for the liquid phase while the pink dotted line terminating in a circle is the locus where the two peaks in the dispersion relation (6) have the same height. The circle denotes the point where the smaller $k$ peak disappears. (b)-(d) $S(k)$ for (b) $\left(\rho_{0} R^{2}, a\right)=(0.8,1.5),(\mathrm{c})(1.5,1.067)$, (d) (2, 0.7). (e)-(g) $\omega(k)$ for (e) $\left(\rho_{0} R^{2}, a\right)=(2,1.5)$, (f) $(2.95,1.067),(\mathrm{g})(3.5,0.7)$. The state points corresponding to the profiles in Fig. 2 are marked with filled squares. 
(a) $(3.6,0.5)$
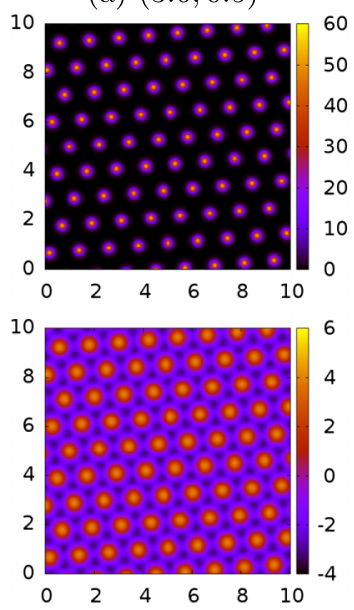

(b) $(3.5,0.76)$
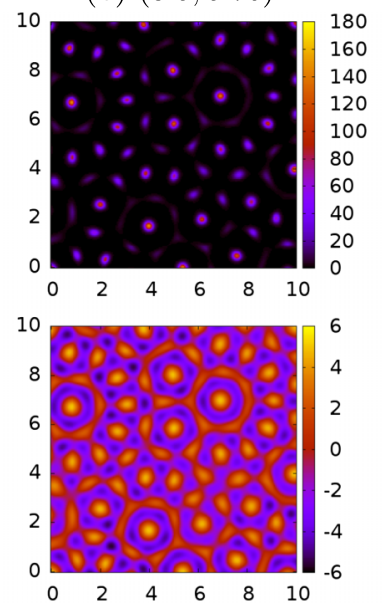

(c) $(4.0,0.8)$
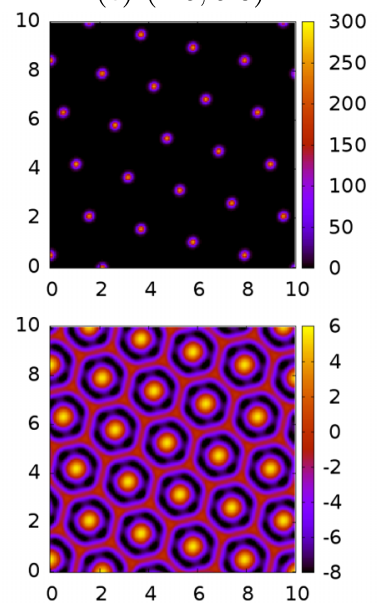

(d) $(2.7,2)$
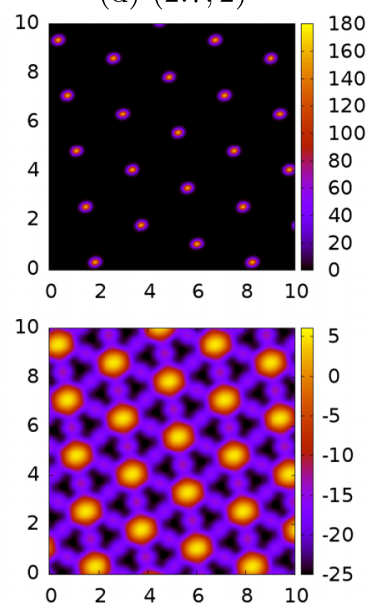

FIG. 2 (color online). Density profiles from DFT showing $R^{2} \rho(\mathbf{r})$ (upper panels) and $\ln \left[R^{2} \rho(\mathbf{r})\right]$ (lower panels). Profiles for (a) $\left(\rho_{0} R^{2}, a\right)=(3.6,0.5)$ (typical of the small length scale crystal B), (b) $(3.6,0.76)$, (c) $(4.0,0.8)$ (both near the transition from crystal $A$ to crystal $B$ ) and (d) $(2.7,2)$ (typical of the large length scale crystal $A$ ). These state points are marked with filled squares in Fig. 1(a). The profiles in (b) show quasicrystalline ordering with numerous defects, while (c) reveals a network of connected density, indicating that the particles in this part of the crystal are fluid, and able to move throughout the system. There are also similar connected fragments in the disordered (b) profile, but because of the disorder, these do not percolate the system.

number $k$ follows $\hat{\rho}(k, t)=\hat{\rho}(k, 0) \exp [\omega(k) t]$, where $\omega(k)$ satisfies the dispersion relation $[46,50]$

$$
\omega(k)=-\Gamma k_{B} T k^{2}\left[1-\rho_{0} \hat{c}(k)\right] .
$$

Here, $\hat{c}(k)$ is the Fourier transform of the pair direct correlation function; within RPA $\hat{c}(k)=-\beta \hat{V}(k)$, where $\hat{V}(k)$ is the Fourier transform of the pair potential in Eq. (1). In an equilibrium fluid the static structure factor $S(k) \equiv$ $\left[1-\rho_{0} \hat{c}(k)\right]^{-1}>0$ for all $k$; such a fluid is therefore stable [51]. Within RPA the two length scales in the pair potential lead, for certain ranges of parameter values, to a static structure factor $S(k)$ with two peaks. Figures 1(b)-1(d) show that as $a$ increases the smaller $k$ peak in $S(k)$ grows and comes to dominate the larger $k$ peak. Figures $1(\mathrm{e})-1(\mathrm{~g})$ show analogous behavior of $\omega(k)$ at several points in or on the boundary of the linearly unstable region $\omega\left(k_{\max }\right)=0$, where $k_{\max }$ is the wave number of the higher peak [blue dashed line in Fig. 1(a)]: as $a$ increases the instability shifts from large $k$ [Fig. 1(g)] to small $k$ [Fig. 1(e)]. The short and long length scales are simultaneously marginally stable at $a=1.067$ and $\rho_{0} R^{2}=2.95$ [Fig. 1(f)]; this point lies on the pink dotted line in Fig. 1(a) corresponding to a pair of equal height peaks in $\omega(k)$. Above (below) this line, the peak at smaller (larger) wave number $k$ is higher, indicating that the longer (shorter) length scale density fluctuations grow the fastest. The black double dotted lines indicate the location of $\omega\left(k_{\max }\right)=0$ for the lower peak in $\omega(k)$. When the system is quenched from a stable liquid state to a state point with density $\rho_{0}$ above the blue dashed line, certain wave numbers will grow as described by $\omega(k)$.

Figure 2(c) shows the density profile of the larger lattice spacing crystal $A$ phase for a state point not far from the transition to the smaller lattice spacing crystal $B$ phase.
However, the panel below displaying $\ln \left[R^{2} \rho(\mathbf{r})\right]$ reveals an interconnected network of channels between the density peaks. The particles contributing to this part of the density profile are fluid in the sense that they can move freely throughout the whole system, unlike the majority of the particles that are located in density peaks at multiply occupied lattice sites. This is the crystal-liquid (CL) state. This state minimizes the free energy for $a>a_{\mathrm{co}}$, where $a_{\mathrm{co}}$ is the value at coexistence. Interfaces between the different phases in Fig. 2 are present whenever these coexist (cf. [52,53]); these will be discussed elsewhere.

To confirm the existence of the CL state we calculate the density profile for a system within a square confining potential $\Phi$ of size $L \times L$ with hard walls, and compare the results with Brownian dynamics (BD) simulations, i.e., simulations of $N$ particles evolving according to Eq. (4). Averaging over the positions of the particles to calculate the density profile, we find remarkably good agreement between the DFT and the BD results (Fig. 3). The resulting system thus consists of two dynamically distinct populations, in contrast to related systems [54-57] in which the dynamics of all the particles are identical. In Fig. 4 we display, for $\beta \mu=39$, the percentage of mobile particles in crystal $A$ as a function of $a$, obtained by integration over all portions of the density profile that are a distance $0.65 R$ away from the center of the density peaks. Particles contributing to this portion of the density are defined to be mobile. For $\beta \mu=0.39$, the two crystal phases coexist at $a_{\text {co }} \approx 0.75$; the proportion of mobile particles increases rapidly as $a \rightarrow a_{\text {co }}$ from above and reaches over $7 \%$ at this value of the chemical potential. In fact, as $a$ is further decreased it is this growing number that triggers the formation of the smaller length scale crystal: these mobile particles freeze to form the extra peaks of crystal $B$. 

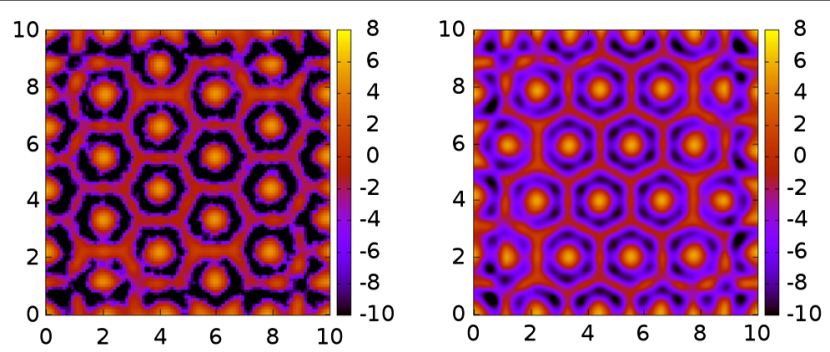

FIG. 3 (color online). $\ln \left[R^{2} \rho(\mathbf{r})\right]$ for a system of $N=600$ particles with $\left(\rho_{0} R^{2}, a\right)=(4.0,0.8)$ confined in a square region of side $L=10 R$ obtained from BD simulations (left panel) and DFT (right panel). The system forms crystal $A$ with a density profile consisting of an array of peaks surrounded by a connected network within which the particles are free to move-the CL state.

Observation of metastable QCs.-A striking aspect of the phase diagram in Fig. 1(a) is that the phase transition between the two different crystal phases (thin red region) is well away from where the two peaks in the dispersion relation have the same height (pink dotted line). A uniform system quenched to the region above the coexistence of the two crystal phases but below this line will initially generate small length scale density fluctuations and the system behaves as if it were going to form crystal $B$. However, the true minimum of the free energy is the larger length scale crystal. Thus, as growing density fluctuations reach the nonlinear regime, the system seeks to go to the longer length scale structure but the smaller length scale imprinted from the linear growth regime leads to frustration. Sometimes the system is able to evolve to the larger length scale crystal; at other times it stays stuck in the metastable small length scale crystal $B$ structure. However, often the system forms a state with density peaks on both length scales, but no long range order. In Fig. 5 we display two rather striking density profiles calculated near state point $2 \mathrm{~b}$ in Fig. 1(a). The upper profile was calculated using Picard iteration starting from random initial conditions. The density profile has many defects, but it has definite quasicrystalline ordering, as can be seen from the corresponding Fourier transform. The lower panels in

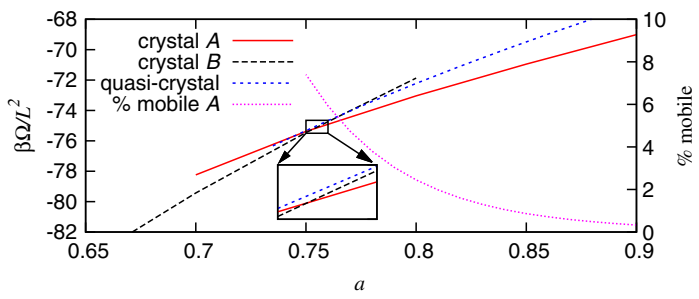

FIG. 4 (color online). Grand potential density for $\beta \mu=39$ as a function of $a$ for the two different crystal structures and the QC solution displayed in Fig. 5. There is a point where all three have almost the same free energy, but the QC solution is never the global minimum of the free energy (see inset). The crystal $A$ phase is of CL type throughout the range of $a$ shown. We also display the percentage of mobile particles in the crystal $A$ phase.
Fig. 5 show a defect-free QC approximant, started from carefully chosen initial conditions. The two wave numbers $k_{1} R=3.2$ and $k_{2} R=6.0$ corresponding to the maxima in $\omega(k)$ are indicated in the Fourier transforms.

The Picard iteration of the Euler-Lagrange equation corresponds to fictitious dynamics since it does not conserve the total number of particles in the system, $N \equiv$ $\int \mathrm{d} \mathbf{r} \rho(\mathbf{r})$. The true dynamics is governed by the DDFT Eq. (5). Evolving this equation is much slower, but in most cases the same qualitative behavior is observed. The Supplemental Material [58] shows time-dependent QC formation obtained using DDFT. The conserved DDFT dynamics does, however, lead to a higher likelihood of getting stuck in the crystal $B$ structure formed in the initial linear growth regime. For $\beta \epsilon=1, R_{S} / R=1.855$ the QCs we find are never the minimum free energy state (Fig. 4). The QC state in Fig. 5 remains stable against small perturbations for $1.77<R_{S} / R<2.18$, but we have not calculated the full phase diagram for $R_{s} / R \neq 1.855$ (at $R_{S} / R=1.885$ the two marginally stable wave numbers [Fig. 1(f)] are very close to the ratio $2 \cos (\pi / 12)=1.93$, favoring 12-fold QCs). We believe it may be possible to use nonlinear dynamics techniques [22] to compute the stability properties of these states by reducing the DDFT description in Eq. (5) to a phase field crystal model, cf. [50,59-62]. We expect that the observed QC formation mechanism (linear growth of one length scale, but nonlinear selection favoring another) may well apply more generally.
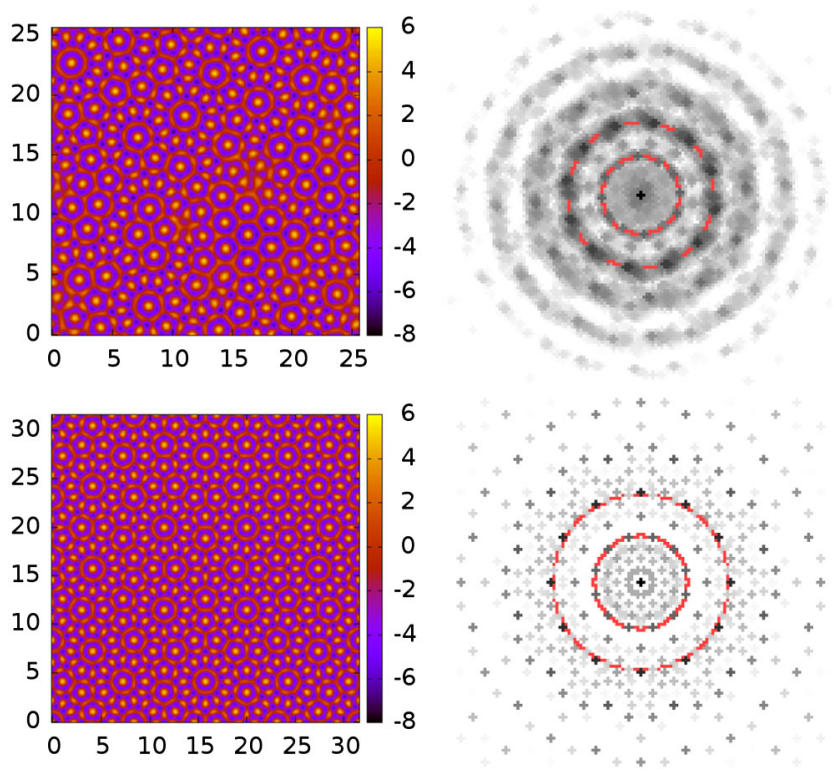

FIG. 5 (color online). Left: $\ln \left[R^{2} \rho(\mathbf{r})\right]$ from DFT, for $\left(\rho_{0} R^{2}, a\right)=(3.5,0.8)$. Right: the corresponding Fourier transforms. The 12-fold symmetry is indicative of QC ordering. The upper profile was obtained from random initial conditions, while the lower one was started from initial conditions with QC symmetry. 
This work was supported in part by the National Science Foundation under Grant No. DMS-1211953 (E. K.). We are grateful for discussions with R. Lifshitz and P. Olmsted.

[1] D. Shechtman, I. Blech, D. Gratias, and J. W. Cahn, Phys. Rev. Lett. 53, 1951 (1984).

[2] A. R. Denton and H. Löwen, Phys. Rev. Lett. 81, 469 (1998).

[3] D. V. Talapin, E. V. Shevchenko, M. I. Bodnarchuk, X. C. Ye, J. Chen, and C. B. Murray, Nature (London) 461, 964 (2009).

[4] C. Xiao, N. Fujita, K. Miyasaka, Y. Sakamoto, and O. Terasaki, Nature (London) 487, 349 (2012).

[5] T. Dotera, Isr. J. Chem. 51, 1197 (2011).

[6] X. Zeng, G. Ungar, Y. Liu, V. Percec, A. E. Dulcey, and J. K. Hobbs, Nature (London) 428, 157 (2004).

[7] S. Fischer, A. Exner, K. Zielske, J. Perlich, S. Deloudi, W. Steurer, P. Lindner, and S. Förster, Proc. Natl. Acad. Sci. U.S.A. 108, 1810 (2011).

[8] A. S. Keys and S. C. Glotzer, Phys. Rev. Lett. 99, 235503 (2007).

[9] C. L. Henley, M. de Boissieu, and W. Steurer, Philos. Mag. 86, 1131 (2006).

[10] H. W. Müller, Phys. Rev. E 49, 1273 (1994).

[11] B. Christiansen, P. Alstrøm, and M. T. Levinsen, Phys. Rev. Lett. 68, 2157 (1992).

[12] B. A. Malomed, A. A. Nepomnyashchiř, and M.I. Tribelskil, Sov. Phys. JETP 69, 388 (1989).

[13] W. S. Edwards and S. Fauve, Phys. Rev. E 47, R788 (1993).

[14] A. Kudrolli, B. Pier, and J.P. Gollub, Physica (Amsterdam) 123D, 99 (1998).

[15] H. Arbell and J. Fineberg, Phys. Rev. E 65, 036224 (2002).

[16] Y. Ding and P. Umbanhowar, Phys. Rev. E 73, 046305 (2006).

[17] W. B. Zhang and J. Viñals, J. Fluid Mech. 336, 301 (1997).

[18] R. Lifshitz and D. M. Petrich, Phys. Rev. Lett. 79, 1261 (1997).

[19] C. M. Topaz, J. Porter, and M. Silber, Phys. Rev. E 70, 066206 (2004).

[20] A. C. Skeldon and G. Guidoboni, SIAM J. Appl. Math. 67, 1064 (2007).

[21] A. M. Rucklidge and M. Silber, SIAM J. Appl. Dyn. Syst. 8, 298 (2009).

[22] A. M. Rucklidge, M. Silber, and A. C. Skeldon, Phys. Rev. Lett. 108, 074504 (2012).

[23] R. Lifshitz and H. Diamant, Philos. Mag. 87, 3021 (2007).

[24] M. Engel and H. R. Trebin, Phys. Rev. Lett. 98, 225505 (2007).

[25] K. Barkan, H. Diamant, and R. Lifshitz, Phys. Rev. B 83, 172201 (2011).

[26] J. Rottler, M. Greenwood, and B. Ziebarth, J. Phys. Condens. Matter 24, 135002 (2012).

[27] C. N. Likos, Phys. Rep. 348, 267 (2001).

[28] J. Dautenhahn and C. K. Hall, Macromolecules 27, 5399 (1994).

[29] C. N. Likos, H. Löwen, M. Watzlawek, B. Abbas, O. Jucknischke, J. Allgaier, and D. Richter, Phys. Rev. Lett. 80, 4450 (1998).
[30] P. G. Bolhuis, A. A. Louis, J.-P. Hansen, and E. J. Meijer, J. Chem. Phys. 114, 4296 (2001).

[31] C. N. Likos and H. M. Harreis, Condens. Matter Phys. 5, 173 (2002).

[32] C. N. Likos, Soft Matter 2, 478 (2006).

[33] D. A. Lenz, R. Blaak, C. N. Likos, and B. M. Mladek, Phys. Rev. Lett. 109, 228301 (2012).

[34] A. Lang, C. N. Likos, M. Watzlawek, and H. Löwen, J. Phys. Condens. Matter 12, 5087 (2000).

[35] A. J. Archer, C. N. Likos, and R. Evans, J. Phys. Condens. Matter 16, L297 (2004).

[36] B. M. Mladek, D. Gottwald, G. Kahl, M. Neumann, and C. N. Likos, J. Phys. Chem. B 111, 12799 (2007).

[37] A. J. Moreno and C. N. Likos, Phys. Rev. Lett. 99, 107801 (2007).

[38] C. N. Likos, B. M. Mladek, D. Gottwald, and G. Kahl, J. Chem. Phys. 126, 224502 (2007).

[39] S. van Teeffelen, A.J. Moreno, and C. N. Likos, Soft Matter 5, 1024 (2009).

[40] A. Nikoubashman, G. Kahl, and C. N. Likos, Soft Matter 8, 4121 (2012).

[41] R. Evans, Adv. Phys. 28, 143 (1979).

[42] R. Evans, in Fundamentals of Inhomogeneous Fluids edited by D. Henderson, (Dekker, New York, 1992).

[43] J. F. Lutsko, Adv. Chem. Phys. 144, 1 (2010).

[44] U. M. B. Marconi and P. Tarazona, J. Chem. Phys. 110, 8032 (1999).

[45] U. M. B. Marconi and P. Tarazona, J. Phys. Condens. Matter 12, A413 (2000).

[46] A. J. Archer and R. Evans, J. Chem. Phys. 121, 4246 (2004).

[47] A. J. Archer and M. Rauscher, J. Phys. A 37, 9325 (2004).

[48] R. Roth, J. Phys. Condens. Matter 22, 063102 (2010).

[49] R. Evans and M. M. Telo da Gama, Mol. Phys. 38, 687 (1979).

[50] A. J. Archer, M. J. Robbins, U. Thiele, and E. Knobloch, Phys. Rev. E 86, 031603 (2012).

[51] J.-P. Hansen and I. R. McDonald, Theory of Simple Liquids (Academic, London, 1986), 2nd ed..

[52] M. Oettel, J. Phys. Condens. Matter 24, 464124 (2012).

[53] B. A. Malomed, A. A. Nepomnyashchy, and M.I. Tribelsky, Phys. Rev. A 42, 7244 (1990).

[54] M. A. Glaser, G. M. Grason, R. D. Kamien, A. Košmrlj, C. D. Santangelo, and P. Ziherl, Europhys. Lett. 78, 46004 (2007).

[55] A. Imperio and L. Reatto, J. Phys. Condens. Matter 16, S3769 (2004).

[56] A. Imperio and L. Reatto, J. Chem. Phys. 124, 164712 (2006).

[57] A. J. Archer, Phys. Rev. E 78, 031402 (2008).

[58] See Supplemental Material at http://link.aps.org/ supplemental/10.1103/PhysRevLett.111.165501 for examples of the dynamics of QC formation.

[59] K. R. Elder, M. Katakowski, M. Haataja, and M. Grant, Phys. Rev. Lett. 88, 245701 (2002).

[60] Z. F. Huang, K. R. Elder, and N. Provatas, Phys. Rev. E 82, 021605 (2010).

[61] S. van Teeffelen, R. Backofen, A. Voigt, and H. Löwen, Phys. Rev. E 79, 051404 (2009).

[62] H. Emmerich, H. Löwen, R. Wittkowski, T. Gruhn, G. I. Tóth, G. Tegze, and L. Gránásy, Adv. Phys. 61, 665 (2012). 